Article

\title{
Optimal Boundary Control of Non-Isothermal Viscous Fluid Flow
}

\author{
Evgenii S. Baranovskii * ${ }^{\mathbb{D}}$, Anastasia A. Domnich ${ }^{\mathbb{D}}$ and Mikhail A. Artemov \\ Department of Applied Mathematics, Informatics and Mechanics, Voronezh State University, \\ 394018 Voronezh, Russia \\ * Correspondence: esbaranovskii@gmail.com
}

Received: 29 June 2019; Accepted: 12 July 2019; Published: 16 July 2019

\begin{abstract}
We study an optimal control problem for the mathematical model that describes steady non-isothermal creeping flows of an incompressible fluid through a locally Lipschitz bounded domain. The control parameters are the pressure and the temperature on the in-flow and out-flow parts of the boundary of the flow domain. We propose the weak formulation of the problem and prove the existence of weak solutions that minimize a given cost functional. It is also shown that the marginal function of this control system is lower semi-continuous.
\end{abstract}

Keywords: non-isothermal flows; creeping flows; viscous fluid; optimal control; boundary control; pressure boundary conditions; weak solution; existence theorem; marginal function

\section{Introduction and Problem Formulation}

In this work, we consider the following optimal control problem for the model describing steady non-isothermal creeping flows of an incompressible fluid through a bounded domain $\Omega \subset \mathbb{R}^{d}$ $(d=2,3)$ with the locally Lipschitz boundary $\partial \Omega$ :

$$
\left\{\begin{array}{l}
-\operatorname{div}[\mu(\theta) \boldsymbol{D}(\boldsymbol{u})]+\nabla p=\boldsymbol{f}(\boldsymbol{x}, \theta) \text { in } \Omega, \\
\operatorname{div} \boldsymbol{u}=0 \text { in } \Omega, \\
(\boldsymbol{u} \cdot \nabla) \theta-\operatorname{div}[\kappa(\theta) \nabla \theta]=\omega(\boldsymbol{x}, \theta) \text { in } \Omega, \\
\boldsymbol{u}=\mathbf{0}, \quad \kappa(\theta) \frac{\partial \theta}{\partial \mathbf{n}}=-\alpha \theta \text { on } \partial \Omega \backslash S, \\
\boldsymbol{u}_{\tau}=\mathbf{0}, \quad p=\pi, \theta=\zeta \text { on } S, \\
(\pi, \zeta) \in \mathbb{U}_{1} \times \mathbb{U}_{2}, \\
J(\boldsymbol{u}, \theta, \pi, \zeta) \rightarrow \min ,
\end{array}\right.
$$

where $u=u(x)$ is the velocity of the fluid at a point $x \in \Omega, \theta=\theta(x)$ is the deviation from the average temperature value, $f(x, \theta)$ denotes the external forces, $\boldsymbol{D}(\boldsymbol{u})$ is the deformation rate tensor, $\boldsymbol{D}(\boldsymbol{u}) \stackrel{\text { def }}{=}\left(\nabla \boldsymbol{u}+(\nabla \boldsymbol{u})^{\mathrm{T}}\right) / 2$, the function $p=p(\boldsymbol{x})$ represents the pressure field, $\mu(\theta)>0$ is the viscosity, $\kappa(\theta)>0$ is the thermal conductivity, $\alpha>0$ is a coefficient characterizing the heat transfer on solid walls of the flow domain, $\omega(x, \theta)$ stands for the heat source intensity, $\mathbf{n}=\mathbf{n}(\boldsymbol{x})$ is the unit outward normal to the surface $\partial \Omega, S$ is a flat (straight for $d=2$ ) portion of $\partial \Omega$ or the union of several such portions. Functions $\zeta: S \rightarrow \mathbb{R}$ and $\pi: S \rightarrow \mathbb{R}$ play the role of controls, $\mathbb{U}_{1} \times \mathbb{U}_{2}$ is the set of admissible controls, while $J=J(u, \theta, \pi, \zeta)$ is a given cost functional. By the symbol $\tau$ we denote the tangential component of a vector, i.e., $\boldsymbol{u}_{\tau} \stackrel{\text { def }}{=} \boldsymbol{u}-(\boldsymbol{u} \cdot \mathbf{n}) \mathbf{n}$. 
The main scope of the present paper is to prove the solvability of problem (1) in the weak formulation. The proof is based on the Galerkin procedure, methods of the topological degree theory, compactness arguments, and the well-known theorem of Krasnoselskii on the continuity of a superposition operator acting in Lebesgue spaces. In addition, for control system (1), we introduce the concept of the marginal function, which shows how the optimal value of the cost functional changes with a change in the set of admissible controls. In this paper, it is proved that the marginal function of (1) is lower semi-continuous.

It should be mentioned that many mathematical works are devoted to optimization and control problems for non-isothermal flows of a viscous fluid. The results from the existing literature mainly deal with the case where the flow occurs inside a bounded domain and external body forces and/or heat sources are used as control parameters (see, for instance, [1-8]). Moreover, the Dirichlet-type boundary control by the velocity field is quite well studied (for details, see References [9-13]). However, for the analysis of real applications, it is very important to consider models of pressure driven flows, which typically occur in control problems for the heat and mass transfer in pipeline networks. For this purpose, following the approach from [14], we employ mixed boundary conditions, including the inhomogeneous Dirichlet condition for the pressure on a portion of the boundary of the flow domain; but at the same time, we formulate the optimal control problem without using the curl operator and boundary conditions associated with this operator. Another distinguishing feature of the present paper is that it takes into account the dependence of both the viscosity and the thermal conductivity coefficient on the temperature. This expands the range of possible applications of our results.

\section{Preliminaries}

In this section, we present some notations and function spaces utilized in the paper.

Let $E_{1}, E_{2}$ be Banach spaces. By $\mathcal{L}\left(E_{1}, E_{2}\right)$ we denote the space of all bounded linear mappings from $E_{1}$ to $E_{2}$. The space $\mathcal{L}\left(E_{1}, E_{2}\right)$ is equipped with the norm

$$
\|A\|_{\mathcal{L}\left(E_{1}, E_{2}\right)} \stackrel{\text { def }}{=} \sup _{\|v\|_{E_{1}} \neq 0} \frac{\|A(v)\|_{E_{2}}}{\|v\|_{E_{1}}} .
$$

The strong (weak) convergence in a Banach space is denoted by $\rightarrow(\rightarrow)$.

Let $\mathcal{U}$ and $\mathcal{W}$ be subsets of a Banach space $E$. By definition, put

$$
d_{E}(\mathcal{U}, \mathcal{W}) \stackrel{\text { def }}{=} \sup _{u \in \mathcal{U}} \inf _{w \in \mathcal{W}}\|u-w\|_{E}
$$

This quantity is termed as the directed Hausdorff distance (or one-sided Hausdorff distance) from the set $\mathcal{U}$ to the set $\mathcal{W}$.

Throughout this paper, boldface symbols denote vector- and matrix-valued quantities. For vectors $x, y \in \mathbb{R}^{d}$ and matrices $\mathbf{X}, \mathbf{Y} \in \mathbb{R}^{d \times d}$, by $x \cdot y$ and $\mathbf{X}: \mathbf{Y}$, we denote the scalar products, respectively:

$$
\boldsymbol{x} \cdot \boldsymbol{y} \stackrel{\text { def }}{=} \sum_{i=1}^{d} x_{i} y_{i}, \quad \mathbf{X}: \mathbf{Y} \stackrel{\text { def }}{=} \sum_{i, j=1}^{d} X_{i j} Y_{i j} .
$$

As usual, $|\cdot|$ denotes the Euclidean norm:

$$
|x| \stackrel{\text { def }}{=}(\boldsymbol{x} \cdot \boldsymbol{x})^{1 / 2}, \quad|\mathbf{X}| \stackrel{\text { def }}{=}(\mathbf{X}: \mathbf{X})^{1 / 2} .
$$

The symbol $\nabla$ stands for the gradient with respect to the variables $x_{1}, \ldots, x_{d}$. The divergence operator is defined as follows: 


$$
\operatorname{div} \boldsymbol{w} \stackrel{\text { def }}{=} \sum_{i=1}^{d} \frac{\partial w_{i}}{\partial x_{i}}, \quad \operatorname{div} Q \stackrel{\text { def }}{=}\left(\sum_{i=1}^{d} \frac{\partial Q_{i 1}}{\partial x_{i}}, \ldots, \sum_{i=1}^{d} \frac{\partial Q_{i d}}{\partial x_{i}}\right)^{\mathrm{T}},
$$

for $\boldsymbol{w}: \mathbb{R}^{d} \rightarrow \mathbb{R}^{d}$ and $Q: \mathbb{R}^{d} \rightarrow \mathbb{R}^{d \times d}$.

We employ the standard notation for Lebesgue spaces, such as $L^{q}(\Omega), L^{q}(\partial \Omega)$, where $q \geq 1$, and the Sobolev space $H^{1}(\Omega) \stackrel{\text { def }}{=} W^{1,2}(\Omega)$. When it comes to classes of $\mathbb{R}^{d}$-valued functions, we use bold face letters, for instance, $L^{q}(\Omega) \stackrel{\text { def }}{=} L^{q}(\Omega)^{d}, H^{1}(\Omega) \stackrel{\text { def }}{=} H^{1}(\Omega)^{d}$, etc.

Recall that the restriction of a function $w \in H^{1}(\Omega)$ to the surface $\partial \Omega$ is defined by the rule $\left.w\right|_{\partial \Omega} \stackrel{\text { def }}{=} \gamma_{\partial \Omega} w$, where $\gamma_{\partial \Omega}: H^{1}(\Omega) \rightarrow L^{q}(\partial \Omega)$ is the trace operator (see, e.g., [15] Section 2.4.2), $q=4$ if $d=3$, and $q$ is an arbitrary number from $[1,+\infty)$ if $d=2$.

Let us introduce two sets:

$$
\begin{aligned}
& \mathbb{V}_{S}(\Omega) \stackrel{\text { def }}{=}\left\{\boldsymbol{v} \in C^{\infty}(\bar{\Omega}): \operatorname{div} \boldsymbol{v}=0,\left.\boldsymbol{v}\right|_{\partial \Omega \backslash S}=\mathbf{0},\left.\boldsymbol{v}_{\tau}\right|_{S}=\mathbf{0}\right\}, \\
& \mathbb{Y}_{S}(\Omega) \stackrel{\text { def }}{=}\left\{\eta \in C^{\infty}(\bar{\Omega}):\left.\eta\right|_{S}=0\right\},
\end{aligned}
$$

and two basic spaces for handling problem (1):

$$
\begin{aligned}
& V_{S}(\Omega) \stackrel{\text { def }}{=} \text { the closure of the set } \mathbb{V}_{S}(\Omega) \text { in the space } H^{1}(\Omega), \\
& Y_{S}(\Omega) \stackrel{\text { def }}{=} \text { the closure of the set } \mathbb{Y}_{S}(\Omega) \text { in the space } H^{1}(\Omega) .
\end{aligned}
$$

In our study, it is convenient to use the following scalar product and norm in $V_{S}(\Omega)$ :

$$
(\boldsymbol{v}, \boldsymbol{w})_{V_{S}(\Omega)} \stackrel{\text { def }}{=} \int_{\Omega} \boldsymbol{D}(\boldsymbol{v}): \boldsymbol{D}(\boldsymbol{w}) d x, \quad\|\boldsymbol{v}\|_{V_{S}(\Omega)} \stackrel{\text { def }}{=}(\boldsymbol{v}, \boldsymbol{v})_{V_{S}(\Omega)}^{1 / 2} .
$$

From ([16] Chap. I, Theorems 2.2 and 2.3) it follows that the scalar product $(\cdot, \cdot)_{V_{S}(\Omega)}$ is well defined and the norm $\|\cdot\|_{V_{S}(\Omega)}$ is equivalent to the standard $H^{1}$-norm if the set $\partial \Omega \backslash S$ has the positive $(d-1)$-dimensional measure.

In the space $Y_{S}(\Omega)$, we introduce the scalar product and the norm as follows:

$$
(\eta, \xi)_{Y_{S}(\Omega)} \stackrel{\text { def }}{=} \int_{\Omega} \nabla \eta \cdot \nabla \xi d x, \quad\|\eta\|_{Y_{S}(\Omega)} \stackrel{\text { def }}{=}(\eta, \eta)_{Y_{S}(\Omega)}^{1 / 2} .
$$

By the Friedrichs inequality (see, e.g., [15] Section 1.1.8, Theorem 1.9), it is easy to show that the scalar product $(\cdot, \cdot)_{Y_{S}(\Omega)}$ is well defined and the norm $\|\cdot\|_{Y_{S}(\Omega)}$ is equivalent to the standard $H^{1}$-norm.

\section{Main Assumptions and Some Examples of Cost Functionals}

Let us assume that the following conditions are fulfilled:

(C1) the inequalities meas $d_{d-1}(S)>0$ and meas $_{d-1}(\partial \Omega \backslash S)>0$ hold, where meas $d_{d-1}(\cdot)$ denotes the Lebesgue $(d-1)$-dimensional measure of a set;

(C2) the functions $\mu: \mathbb{R} \rightarrow \mathbb{R}$ and $\kappa: \mathbb{R} \rightarrow \mathbb{R}$ are continuous and there exist constants $\mu_{i}, \kappa_{i}, i=0,1$, such that $0<\mu_{0} \leq \mu(y) \leq \mu_{1}, 0<\kappa_{0} \leq \kappa(y) \leq \kappa_{1}$ for every $y \in \mathbb{R}$;

(C3) the functions $f_{i}(\cdot, y): \Omega \rightarrow \mathbb{R}, i=1, \ldots, d$, and $\omega(\cdot, y): \Omega \rightarrow \mathbb{R}$ are measurable for every $y \in \mathbb{R}$;

(C4) the functions $f_{i}(\boldsymbol{x}, \cdot): \mathbb{R} \rightarrow \mathbb{R}, i=1, \ldots, d$, and $\omega(\boldsymbol{x}, \cdot): \mathbb{R} \rightarrow \mathbb{R}$ are continuous for almost every $x \in \Omega$;

(C5) there exist functions $g_{0}$ and $\omega_{0}$ from the space $L^{2}(\Omega)$ and a positive constant $M$ such that

$$
|f(x, y)| \leq g_{0}(x)+M|y|^{2}, \quad|\omega(\boldsymbol{x}, y)| \leq \omega_{0}(\boldsymbol{x}),
$$

for every $y \in \mathbb{R}$ and for almost every $x \in \Omega$; 
(C6) the set $\mathbb{U}_{1}$ is sequentially weakly closed in the space $L^{2}(S)$;

(C7) the set $\mathbb{U}_{2}$ is closed in the space $L^{2}(S)$ and $0 \in \mathbb{U}_{2}$;

(C8) the functional $J: V_{S}(\Omega) \times H^{1}(\Omega) \times L^{2}(S) \times L^{2}(S) \rightarrow \mathbb{R}$ is lower weakly semi-continuous; that is, for any sequence $\left\{\left(\boldsymbol{u}^{n}, \theta^{n}, \pi^{n}, \zeta^{n}\right)\right\}_{n=1}^{\infty}$ such that $\boldsymbol{u}^{n} \rightarrow \boldsymbol{u}^{0}$ weakly in $\boldsymbol{V}_{S}(\Omega), \theta^{n} \rightarrow \theta^{0}$ weakly in $H^{1}(\Omega), \pi^{n} \rightarrow \pi^{0}$ weakly in $L^{2}(S)$, and $\zeta^{n} \rightarrow \zeta^{0}$ weakly in $L^{2}(S)$ as $n \rightarrow \infty$, we have

$$
J\left(\boldsymbol{u}^{0}, \theta^{0}, \pi^{0}, \zeta^{0}\right) \leq \liminf _{n \rightarrow \infty} J\left(\boldsymbol{u}^{n}, \theta^{n}, \pi^{n}, \zeta^{n}\right) ;
$$

(C9) the coercivity condition [17] holds for $J$; that is, for every $R>0$ the set

$$
\mathcal{G}_{R} \stackrel{\text { def }}{=}\left\{(\boldsymbol{u}, \theta, \pi, \zeta) \in \boldsymbol{V}_{S}(\Omega) \times H^{1}(\Omega) \times L^{2}(S) \times L^{2}(S): J(\boldsymbol{u}, \theta, \pi, \zeta) \leq R\right\}
$$

is bounded in $\boldsymbol{V}_{S}(\Omega) \times H^{1}(\Omega) \times L^{2}(S) \times L^{2}(S)$.

Here, we also give two examples of cost functionals satisfying conditions (C8) and (C9):

$$
\begin{aligned}
J=J_{1}(\boldsymbol{u}, \theta, \pi, \zeta) \stackrel{\text { def }}{=} & \lambda_{1} \int_{\Omega}\left|\boldsymbol{u}-\boldsymbol{u}_{0}\right|^{2} d x+\lambda_{2} \sum_{i=1}^{d} \int_{\Omega}\left|\frac{\partial\left(\boldsymbol{u}-\boldsymbol{u}_{0}\right)}{\partial x_{i}}\right|^{2} d x \\
& +\lambda_{3} \int_{\Omega}\left|\theta-\theta_{0}\right|^{2} d x+\lambda_{4} \sum_{i=1}^{d} \int_{\Omega}\left|\frac{\partial\left(\theta-\theta_{0}\right)}{\partial x_{i}}\right|^{2} d x \\
& +\lambda_{5} \int_{S}|\pi|^{2} d \sigma+\lambda_{6} \int_{S}|\zeta|^{2} d \sigma \\
J=J_{2}(\boldsymbol{u}, \theta, \pi, \zeta) \stackrel{\text { def }}{=} & \lambda_{1} \int_{\Omega}|\boldsymbol{D}(\boldsymbol{u})|^{2} d x+\lambda_{2} \int_{\Omega}|\nabla \theta|^{2} d x+\lambda_{3} \int_{\partial \Omega \backslash S}|\theta|^{2} d \sigma \\
& +\lambda_{4} \int_{S}\left|\pi-\pi_{0}\right|^{2} d \sigma+\lambda_{5} \int_{S}\left|\zeta-\zeta_{0}\right|^{2} d \sigma,
\end{aligned}
$$

where $\boldsymbol{u}_{0}, \theta_{0}, \pi_{0}, \zeta_{0}$ are given functions, and $\lambda_{1}, \lambda_{2}, \ldots, \lambda_{6}$ are positive parameters (weight coefficients).

\section{Weak Formulation of Problem (1) and Marginal Function}

Definition 1. We shall say that $(\boldsymbol{u}, \theta, \pi, \zeta) \in \boldsymbol{V}_{S}(\Omega) \times H^{1}(\Omega) \times L^{2}(S) \times L^{2}(S)$ is an admissible quadruplet to problem (1) if $(\pi, \zeta) \in \mathbb{U}_{1} \times \mathbb{U}_{2},\left.\theta\right|_{S}=\zeta$, and

$$
\begin{gathered}
\int_{\Omega} \mu(\theta) \boldsymbol{D}(\boldsymbol{u}): \boldsymbol{D}(\boldsymbol{v}) d x+\int_{S} \pi(\boldsymbol{v} \cdot \mathbf{n}) d \sigma=\int_{\Omega} \boldsymbol{f}(\boldsymbol{x}, \theta) \cdot \boldsymbol{v} d x, \\
\sum_{i=1}^{d} \int_{\Omega} u_{i} \frac{\partial \theta}{\partial x_{i}} \eta \boldsymbol{d} \boldsymbol{x}+\int_{\Omega} \kappa(\theta) \nabla \theta \cdot \nabla \eta \boldsymbol{d} x+\alpha \int_{\partial \Omega \backslash S} \theta \eta d \sigma=\int_{\Omega} \omega(\boldsymbol{x}, \theta) \eta \boldsymbol{d} \boldsymbol{x}
\end{gathered}
$$

for any vector-valued function $v \in \boldsymbol{V}_{S}(\Omega)$ and function $\eta \in Y_{S}(\Omega)$.

The following lemma shows that relations (2) and (3) are natural in the weak formulation of problem (1).

Lemma 1. Suppose $(\boldsymbol{u}, \theta, p)$ is a classic solution of boundary-value problem $(1)_{1}-(1)_{5}$, then equalities (2) and (3) hold.

Proof. Let us fix an arbitrary vector-valued function $v \in V_{S}(\Omega)$. On taking the scalar product of both the left-hand and right-hand sides of equality $(1)_{1}$ with $v$ and integrating over the domain $\Omega$, we obtain

$$
-\underbrace{\int_{\Omega} \operatorname{div}[\mu(\theta) \boldsymbol{D}(\boldsymbol{u})] \cdot \boldsymbol{v} d x}_{I_{1}}+\underbrace{\int_{\Omega} \nabla p \cdot \boldsymbol{v} d x}_{I_{2}}=\int_{\Omega} f(x, \theta) \cdot \boldsymbol{v} d x .
$$


By using integration by parts, one can show that

$$
I_{1}=\int_{\partial \Omega}[\mu(\theta) \boldsymbol{D}(\boldsymbol{u}) \mathbf{n}] \cdot \boldsymbol{v} d \sigma-\int_{\Omega} \mu(\theta) \boldsymbol{D}(\boldsymbol{u}): \boldsymbol{D}(\boldsymbol{v}) d \boldsymbol{x},
$$

from which, bearing in mind that $v=(v \cdot \mathbf{n}) \mathbf{n}$ on $S$ and $v=\mathbf{0}$ on $\partial \Omega \backslash S$, we derive

$$
I_{1}=\int_{S} \mu(\theta)([\boldsymbol{D}(\boldsymbol{u}) \mathbf{n}] \cdot \mathbf{n})(\boldsymbol{v} \cdot \mathbf{n}) d \sigma-\int_{\Omega} \mu(\theta) \boldsymbol{D}(\boldsymbol{u}): \boldsymbol{D}(\boldsymbol{v}) d x .
$$

Note that

$$
[\boldsymbol{D}(\boldsymbol{u}) \mathbf{n}] \cdot \mathbf{n}=\frac{\partial(\boldsymbol{u} \cdot \mathbf{n})}{\partial \mathbf{n}} \text { on } S .
$$

Since

$$
\operatorname{div} \boldsymbol{u}=0 \text { in } \Omega, \quad \boldsymbol{u}_{\tau}=0 \text { on } S,
$$

we see that

$$
\frac{\partial(\boldsymbol{u} \cdot \mathbf{n})}{\partial \mathbf{n}}=0 \text { on } S
$$

and hence,

$$
[\boldsymbol{D}(\boldsymbol{u}) \mathbf{n}] \cdot \mathbf{n}=0 \text { on } S .
$$

Substituting the last relation into (5), we obtain, obviously,

$$
I_{1}=-\int_{\Omega} \mu(\theta) \boldsymbol{D}(u): D(v) d x
$$

To handle the term $I_{2}$, we again use integration by parts and get

$$
I_{2}=\int_{\partial \Omega} p(v \cdot \mathbf{n}) d \sigma-\int_{\Omega} p \operatorname{div} v d x .
$$

Since

$$
\operatorname{div} v=0 \text { in } \Omega, \quad v=\mathbf{0} \text { on } \Omega \backslash S, \quad p=\pi \text { on } S,
$$

from (7) it follows that

$$
I_{2}=\int_{S} \pi(\boldsymbol{v} \cdot \mathbf{n}) d \sigma
$$

Finally, by substituting (6) and (8) into equality (4), we arrive at (2).

Next, we take the scalar product in $L^{2}(\Omega)$ of each term from $(1)_{3}$ with a function $\eta \in Y_{S}(\Omega)$ and get

$$
\sum_{i=1}^{d} \int_{\Omega} u_{i} \frac{\partial \theta}{\partial x_{i}} \eta d x-\underbrace{\int_{\Omega} \operatorname{div}[\kappa(\theta) \nabla \theta] \eta d x}_{I_{3}}=\int_{\Omega} \omega(x, \theta) \eta d x .
$$

Integrating by parts the term $I_{3}$, we can rewrite the last equality as follows:

$$
\sum_{i=1}^{d} \int_{\Omega} u_{i} \frac{\partial \theta}{\partial x_{i}} \eta \boldsymbol{d} x-\int_{\partial \Omega} \kappa(\theta) \frac{\partial \theta}{\partial \mathbf{n}} \eta d \sigma+\int_{\Omega} \kappa(\theta) \nabla \theta \cdot \nabla \eta \boldsymbol{d} x=\int_{\Omega} \omega(\boldsymbol{x}, \theta) \eta \boldsymbol{d} \boldsymbol{x} .
$$

Taking into account the boundary conditions

$$
\kappa(\theta) \frac{\partial \theta}{\partial \mathbf{n}}=-\alpha \theta \text { on } \partial \Omega \backslash S, \quad \eta=0 \text { on } S,
$$

we arrive at equality (3). This finishes the proof of Lemma 1. 
We are now ready to accurately define the concept of solutions to the considered optimal control problem. Let $\mathfrak{M}\left(\mathbb{U}_{1}, \mathbb{U}_{2}\right)$ be the set of admissible quadruplets to problem (1).

Definition 2. By a solution of problem (1) we mean a quadruplet $\left(\boldsymbol{u}_{*}, \theta_{*}, \pi_{*}, \zeta_{*}\right) \in \mathfrak{M}\left(\mathbb{U}_{1}, \mathbb{U}_{2}\right)$ at which the functional $J$ attains the minimum:

$$
J\left(\boldsymbol{u}_{*}, \theta_{*}, \pi_{*}, \zeta_{*}\right)=\inf _{(\boldsymbol{u}, \theta, \pi, \zeta) \in \mathfrak{M}\left(\mathbb{U}_{1}, \mathbb{U}_{2}\right)} J(\boldsymbol{u}, \theta, \pi, \zeta) .
$$
define

By $\mathfrak{M}_{\text {opt }}\left(\mathbb{U}_{1}, \mathbb{U}_{2}\right)$ denote the set of solutions to problem (1). Assuming that $\mathfrak{M}_{\text {opt }}\left(\mathbb{U}_{1}, \mathbb{U}_{2}\right) \neq \varnothing$, we

$$
\Phi\left(\mathbb{U}_{1}, \mathbb{U}_{2}\right) \stackrel{\text { def }}{=} J\left(\boldsymbol{u}_{*}, \theta_{*}, \pi_{*}, \zeta_{*}\right),
$$

where $\left(\boldsymbol{u}_{*}, \theta_{*}, \pi_{*}, \zeta_{*}\right)$ is a quadruplet that belongs to the set $\mathfrak{M}_{\mathrm{opt}}\left(\mathbb{U}_{1}, \mathbb{U}_{2}\right)$. It is obvious that the value of $\Phi\left(\mathbb{U}_{1}, \mathbb{U}_{2}\right)$ does not depend on the choice of an element from the set $\mathfrak{M}_{\text {opt }}\left(\mathbb{U}_{1}, \mathbb{U}_{2}\right)$ in the right-hand sides of (9).

Definition 3. The function $\Phi$ that is defined by equality (9) is called the marginal function of control system (1).

\section{Main Results}

The main results of the present work are summarized as follows:

Theorem 1. Suppose conditions (C1)-(C9) hold. Then:

(a) optimal control problem (1) has at least one solution;

(b) the marginal function $\Phi$ is lower semi-continuous in the following sense:

if $\mathfrak{M}_{\mathrm{opt}}\left(\mathbb{U}_{1}^{n}, \mathbb{U}_{2}^{n}\right) \neq \varnothing$, for any $n \in \mathbb{N}$, and

$$
\lim _{n \rightarrow \infty} d_{L^{2}(S)}\left(\mathbb{U}_{1}^{n}, \mathbb{U}_{1}\right)=0, \quad \lim _{n \rightarrow \infty} d_{L^{2}(S)}\left(\mathbb{U}_{2}^{n}, \mathbb{U}_{2}\right)=0,
$$

then

$$
\Phi\left(\mathbb{U}_{1}, \mathbb{U}_{2}\right) \leq \liminf _{n \rightarrow \infty} \Phi\left(\mathbb{U}_{1}^{n}, \mathbb{U}_{2}^{n}\right)
$$

The proof of this theorem is given in Section 7.

\section{Auxiliary Propositions}

For the reader's convenience, we state here some preparatory results on which the proof of Theorem 1 is based.

Proposition 1. Suppose $\mathfrak{B}_{r} \stackrel{\text { def }}{=}\left\{\boldsymbol{a} \in \mathbb{R}^{N}:|\boldsymbol{a}|<r\right\}$ and $\boldsymbol{F}: \overline{\mathfrak{B}}_{r} \times[0,1] \rightarrow \mathbb{R}^{N}$ is a continuous mapping such that

(i) $\boldsymbol{F}(\boldsymbol{a}, \lambda) \neq \mathbf{0}$ for any pair $(\boldsymbol{a}, \lambda) \in \partial \mathfrak{B}_{r} \times[0,1]$;

(ii) $\boldsymbol{F}(\cdot, 0): \overline{\mathfrak{B}}_{r} \rightarrow \mathbb{R}^{N}$ is an odd mapping; that is, $\boldsymbol{F}(-\boldsymbol{a}, 0)=-\boldsymbol{F}(\boldsymbol{a}, 0)$ for any vector $\boldsymbol{a} \in \overline{\mathfrak{B}}_{r}$.

Then, for any $\lambda \in[0,1]$, there exists a vector $\boldsymbol{a}_{\lambda} \in \mathfrak{B}_{r}$ such that $\boldsymbol{F}\left(\boldsymbol{a}_{\lambda}, \lambda\right)=\mathbf{0}$; in other words, the equation $\boldsymbol{F}(\boldsymbol{a}, \lambda)=\mathbf{0}$ is solvable with respect to $\boldsymbol{a}$ in the ball $\mathfrak{B}_{r}$.

Proof. Employing the homotopy invariance property of Brouwer's degree and condition (i), one can show that $\operatorname{deg}\left(\boldsymbol{F}(\cdot, \lambda), \mathfrak{B}_{r}, \mathbf{0}\right)$ does not depend on $\lambda \in[0,1]$ and, consequently,

$$
\operatorname{deg}\left(\boldsymbol{F}(\cdot, \lambda), \mathfrak{B}_{r}, \mathbf{0}\right)=\operatorname{deg}\left(\boldsymbol{F}(\cdot, 0), \mathfrak{B}_{r}, \mathbf{0}\right),
$$


for any $\lambda \in[0,1]$. Besides, since $\boldsymbol{F}(\cdot, 0): \overline{\mathfrak{B}}_{r} \rightarrow \mathbb{R}^{N}$ is an odd mapping, we see that $\operatorname{deg}\left(\boldsymbol{F}(\cdot, 0), \mathfrak{B}_{r}, \mathbf{0}\right)$ is an odd number. This follows from Borsuk's theorem (see, e.g., [18] Chap. 1, Theorem 1.2.11). Thereby, for any $\lambda \in[0,1]$,

$$
\operatorname{deg}\left(\boldsymbol{F}(\cdot, \lambda), \mathfrak{B}_{r}, \mathbf{0}\right) \neq 0 .
$$

Then, by [18] (Chap. 1, Theorem 1.2.6), we deduce that the equation $\boldsymbol{F}(\boldsymbol{a}, \lambda)=\mathbf{0}$ has at least one solution $\boldsymbol{a}_{\lambda} \in \mathfrak{B}_{r}$.

Proposition 2. Suppose $G \subset \mathbb{R}^{N}$ is a Lebesgue measurable set and a given function $\varphi: G \times \mathbb{R} \rightarrow \mathbb{R}$ satisfies the following conditions:

- the function $\varphi(\cdot, y): G \rightarrow \mathbb{R}$ is measurable for every $y \in \mathbb{R}$;

- the function $\varphi(\boldsymbol{x}, \cdot): \mathbb{R} \rightarrow \mathbb{R}$ is continuous for almost every $x \in G$;

- there exist constants $q_{1} \geq 1, q_{2} \geq 1, K>0$ and a function $\phi_{0} \in L^{q_{2}}(G)$ such that

$$
|\varphi(x, y)| \leq \phi_{0}(x)+K|y|^{q_{1} / q_{2}}
$$

for every $y \in \mathbb{R}$ and for almost every $x \in G$.

Then, the superposition operator $T_{\varphi}$ defined by

$$
T_{\varphi}: L^{q_{1}}(G) \rightarrow L^{q_{2}}(G), \quad T_{\varphi}[w](x) \stackrel{\text { def }}{=} \varphi(x, w(x))
$$

is a bounded and continuous mapping.

This proposition was proved by Krasnoselskii (see [19] Chap. 1).

\section{Proof of Theorem 1}

Let us prove the existence result (a). Our first step is to show that $\mathfrak{M}\left(\mathbb{U}_{1}, \mathbb{U}_{2}\right) \neq \varnothing$.

Fix a pair $\left(\pi^{0}, 0\right) \in \mathbb{U}_{1} \times \mathbb{U}_{2}$. Let $\left\{\boldsymbol{v}^{j}\right\}_{j=1}^{\infty} \subset \mathbb{V}_{S}(\Omega)$ be an orthonormal basis of the space $V_{S}(\Omega)$, $\left\{\eta^{j}\right\}_{j=1}^{\infty} \subset \mathbb{Y}_{S}(\Omega)$ an orthonormal basis of the space $Y_{S}(\Omega)$, and $m$ an arbitrary fixed integer.

Consider the following one-parameter problem in space $\mathbb{R}^{2 m}$ :

Find a vector $\left(a_{m 1}, \ldots, a_{m m}, b_{m 1}, \ldots, b_{m m}\right)^{\mathrm{T}} \in \mathbb{R}^{2 m}$ such that

$$
\begin{aligned}
\int_{\Omega} \mu\left(\lambda \theta^{m}\right) \boldsymbol{D}\left(\boldsymbol{u}^{m}\right): \boldsymbol{D}\left(\boldsymbol{v}^{j}\right) d x+\lambda \int_{S} \pi^{0}\left(\boldsymbol{v}^{j} \cdot \mathbf{n}\right) d \sigma & =\lambda \int_{\Omega} f\left(x, \theta^{m}\right) \cdot \boldsymbol{v}^{j} d x, \quad j=1, \ldots, m, \\
\lambda \sum_{i=1}^{d} \int_{\Omega} u_{i}^{m} \frac{\partial \theta^{m}}{\partial x_{i}} \eta^{j} d x+\int_{\Omega} \kappa\left(\lambda \theta^{m}\right) \nabla \theta^{m} \cdot \nabla \eta^{j} d x+\lambda \alpha \int_{\partial \Omega \backslash S} \theta^{m} \eta^{j} d \sigma & \\
& =\lambda \int_{\Omega} \omega\left(x, \theta^{m}\right) \eta^{j} d x, \quad j=1, \ldots, m,
\end{aligned}
$$

where $\boldsymbol{u}^{m}$ and $\theta^{m}$ are defined as follows:

$$
\boldsymbol{u}^{m} \stackrel{\text { deff }}{=} \sum_{j=1}^{m} a_{m j} \boldsymbol{v}^{j}, \quad \theta^{m} \stackrel{\text { def }}{=} \sum_{j=1}^{m} b_{m j} \eta^{j},
$$

and $\lambda$ is a parameter, $\lambda \in[0,1]$.

We shall establish a priori estimates of solutions to problem (11)-(13). Suppose a vector $\left(a_{m 1}, \ldots, a_{m m}, b_{m 1}, \ldots, b_{m m}\right)^{\mathrm{T}}$ satisfies (11)-(13). Let us multiply (11) by $a_{m j}$ and add the obtained equalities for $j=1, \ldots, m$. This yields

$$
\int_{\Omega} \mu\left(\lambda \theta^{m}\right)\left|\boldsymbol{D}\left(\boldsymbol{u}^{m}\right)\right|^{2} d x+\lambda \int_{S} \pi^{0}\left(\boldsymbol{u}^{m} \cdot \mathbf{n}\right) d \sigma=\lambda \int_{\Omega} f\left(x, \theta^{m}\right) \cdot \boldsymbol{u}^{m} d x
$$


Then, using conditions (C2) and (C5), the Cauchy-Bunyakovsky-Schwarz inequality, and $0 \leq \lambda \leq 1$, we derive

$$
\begin{aligned}
\mu_{0}\left\|\boldsymbol{u}^{m}\right\|_{\boldsymbol{V}_{S}(\Omega)}^{2}= & \mu_{0} \int_{\Omega}\left|\boldsymbol{D}\left(\boldsymbol{u}^{m}\right)\right|^{2} \boldsymbol{d} \boldsymbol{x} \\
\leq & \int_{\Omega} \mu\left(\lambda \theta^{m}\right)\left|\boldsymbol{D}\left(\boldsymbol{u}^{m}\right)\right|^{2} \boldsymbol{d} \boldsymbol{x} \\
= & -\lambda \int_{S} \pi^{0}\left(\boldsymbol{u}^{m} \cdot \mathbf{n}\right) d \sigma+\lambda \int_{\Omega} f\left(\boldsymbol{x}, \theta^{m}\right) \cdot \boldsymbol{u}^{m} \boldsymbol{d x} \\
\leq & \left(\int_{S}\left|\pi^{0}\right|^{2} d \sigma\right)^{1 / 2}\left(\int_{S}\left|\boldsymbol{u}^{m}\right|^{2} d \sigma\right)^{1 / 2}+\left(\int_{\Omega}\left|g_{0}\right|^{2} \boldsymbol{d} \boldsymbol{x}\right)^{1 / 2}\left(\int_{\Omega}\left|\boldsymbol{u}^{m}\right|^{2} \boldsymbol{d} \boldsymbol{x}\right)^{1 / 2} \\
& +M\left(\int_{\Omega}\left|\theta^{m}\right|^{4} d \boldsymbol{x}\right)^{1 / 2}\left(\int_{\Omega}\left|\boldsymbol{u}^{m}\right|^{2} \boldsymbol{d x}\right)^{1 / 2} \\
= & \left\|\pi^{0}\right\|_{L^{2}(S)}\left\|\boldsymbol{u}^{m}\right\|_{L^{2}(S)}+\left\|g_{0}\right\|_{L^{2}(\Omega)}\left\|\boldsymbol{u}^{m}\right\|_{L^{2}(\Omega)}+M\left\|\theta^{m}\right\|_{L^{4}(\Omega)}^{2}\left\|\boldsymbol{u}^{m}\right\|_{L^{2}(\Omega)} .
\end{aligned}
$$

Note that

$$
\begin{aligned}
\left\|\boldsymbol{u}^{m}\right\|_{\mathbf{L}^{2}(S)} & \leq\left\|\gamma_{S}\right\|_{\mathcal{L}\left(\boldsymbol{V}_{S}(\Omega), \boldsymbol{L}^{2}(S)\right)}\left\|\boldsymbol{u}^{m}\right\|_{\boldsymbol{V}_{S}(\Omega)} \\
\left\|\boldsymbol{u}^{m}\right\|_{L^{2}(\Omega)} & \leq\|\mathcal{I}\|_{\mathcal{L}\left(V_{S}(\Omega), \boldsymbol{L}^{2}(\Omega)\right)}\left\|\boldsymbol{u}^{m}\right\|_{V_{S}(\Omega)} \\
\left\|\theta^{m}\right\|_{L^{4}(\Omega)} & \leq\|\mathcal{I}\|_{\mathcal{L}\left(Y_{S}(\Omega), L^{4}(\Omega)\right)}\left\|\theta^{m}\right\|_{\gamma_{S}(\Omega)}
\end{aligned}
$$

where $\gamma_{S}$ is the trace operator, the symbol $\mathcal{I}$ stands for the identity mapping. Combining (14) with (15)-(17), we get

$$
\begin{aligned}
\mu_{0}\left\|\boldsymbol{u}^{m}\right\|_{\boldsymbol{V}_{S}(\Omega)}^{2} \leq & \left\|\pi^{0}\right\|_{L^{2}(S)}\left\|\gamma_{S}\right\|_{\mathcal{L}\left(\boldsymbol{V}_{S}(\Omega), \boldsymbol{L}^{2}(S)\right)}\left\|\boldsymbol{u}^{m}\right\|_{\boldsymbol{V}_{S}(\Omega)} \\
& +\left\|g_{0}\right\|_{L^{2}(\Omega)}\|\mathcal{I}\|_{\mathcal{L}\left(\boldsymbol{V}_{S}(\Omega), \boldsymbol{L}^{2}(\Omega)\right)}\left\|\boldsymbol{u}^{m}\right\|_{\boldsymbol{V}_{S}(\Omega)} \\
& +M\|\mathcal{I}\|_{\mathcal{L}\left(Y_{S}(\Omega), L^{4}(\Omega)\right)}^{2}\|\mathcal{I}\|_{\mathcal{L}\left(\boldsymbol{V}_{S}(\Omega), \boldsymbol{L}^{2}(\Omega)\right)}\left\|\theta^{m}\right\|_{Y_{S}(\Omega)}^{2}\left\|\boldsymbol{u}^{m}\right\|_{\boldsymbol{V}_{S}(\Omega)}
\end{aligned}
$$

It immediately follows that

$$
\begin{aligned}
\left\|\boldsymbol{u}^{m}\right\|_{\boldsymbol{V}_{S}(\Omega)} \leq & \mu_{0}^{-1}\left\|\pi^{0}\right\|_{L^{2}(S)}\left\|\gamma_{S}\right\|_{\mathcal{L}\left(\boldsymbol{V}_{\mathcal{S}}(\Omega), \boldsymbol{L}^{2}(S)\right)}+\mu_{0}^{-1}\left\|g_{0}\right\|_{L^{2}(\Omega)}\|\mathcal{I}\|_{\mathcal{L}\left(\boldsymbol{V}_{S}(\Omega), \boldsymbol{L}^{2}(\Omega)\right)} \\
& +M \mu_{0}^{-1}\|\mathcal{I}\|_{\mathcal{L}\left(Y_{S}(\Omega), L^{4}(\Omega)\right)}^{2}\|\mathcal{I}\|_{\mathcal{L}\left(V_{S}(\Omega), L^{2}(\Omega)\right)}\left\|\theta^{m}\right\|_{Y_{S}(\Omega)}^{2} .
\end{aligned}
$$

Now multiply (12) by $b_{m j}$ and add the results for $j=1, \ldots, m$; this gives

$$
\lambda \underbrace{\sum_{i=1}^{d} \int_{\Omega} u_{i}^{m} \frac{\partial \theta^{m}}{\partial x_{i}} \theta^{m} d x}_{I_{4}}+\int_{\Omega} \kappa\left(\lambda \theta^{m}\right)\left|\nabla \theta^{m}\right|^{2} d x+\lambda \alpha \int_{\partial \Omega \backslash S}\left|\theta^{m}\right|^{2} d \sigma=\lambda \int_{\Omega} \omega\left(\boldsymbol{x}, \theta^{m}\right) \theta^{m} d \boldsymbol{x} .
$$

Using the integration by parts formula, it is easy to show that the term $I_{4}$ vanishes. Indeed,

$$
\begin{aligned}
I_{4} & =\frac{1}{2} \sum_{i=1}^{d} \int_{\Omega} u_{i}^{m} \frac{\partial\left|\theta^{m}\right|^{2}}{\partial x_{i}} d x \\
& =\frac{1}{2} \sum_{i=1}^{d} \int_{\partial \Omega} u_{i}^{m} n_{i}\left|\theta^{m}\right|^{2} d \sigma-\frac{1}{2} \sum_{i=1}^{d} \int_{\Omega} \frac{\partial u_{i}^{m}}{\partial x_{i}}\left|\theta^{m}\right|^{2} d x \\
& =\frac{1}{2} \int_{S}\left(\boldsymbol{u}^{m} \cdot \mathbf{n}\right) \underbrace{\left|\theta^{m}\right|^{2}}_{=0} d \sigma+\frac{1}{2} \int_{\partial \Omega \backslash S}(\underbrace{u^{m} \cdot \mathbf{n}}_{=0})\left|\theta^{m}\right|^{2} d \sigma-\frac{1}{2} \int_{\Omega} \underbrace{\operatorname{div} \boldsymbol{u}^{m}}_{=0}\left|\theta^{m}\right|^{2} d x \\
& =0 .
\end{aligned}
$$


Therefore, equality (19) can be rewritten as follows:

$$
\int_{\Omega} \kappa\left(\lambda \theta^{m}\right)\left|\nabla \theta^{m}\right|^{2} d x=-\lambda \alpha \int_{\partial \Omega \backslash S}\left|\theta^{m}\right|^{2} d \sigma+\lambda \int_{\Omega} \omega\left(x, \theta^{m}\right) \theta^{m} d x,
$$

from which, by using conditions (C2) and (C5), the Cauchy-Bunyakovsky-Schwarz inequality, and $0 \leq \lambda \leq 1$, we derive

$$
\begin{aligned}
\kappa_{0}\left\|\theta^{m}\right\|_{Y_{S}(\Omega)}^{2} & =\kappa_{0} \int_{\Omega}\left|\nabla \theta^{m}\right|^{2} d x \\
& \leq \int_{\Omega} \kappa\left(\lambda \theta^{m}\right)\left|\nabla \theta^{m}\right|^{2} d x \\
& =-\lambda \alpha \int_{\partial \Omega \backslash S}\left|\theta^{m}\right|^{2} d \sigma+\lambda \int_{\Omega} \omega\left(x, \theta^{m}\right) \theta^{m} d x \\
& \leq\left(\int_{\Omega}\left|\omega_{0}\right|^{2} d x\right)^{1 / 2}\left(\int_{\Omega}\left|\theta^{m}\right|^{2} d x\right)^{1 / 2} \\
& =\left\|\omega_{0}\right\|_{L^{2}(\Omega)}\left\|\theta^{m}\right\|_{L^{2}(\Omega)} \\
& \leq\left\|\omega_{0}\right\|_{L^{2}(\Omega)}\|\mathcal{I}\|_{\mathcal{L}\left(Y_{S}(\Omega), L^{2}(\Omega)\right)}\left\|\theta^{m}\right\|_{Y_{S}(\Omega)}
\end{aligned}
$$

whence

$$
\left\|\theta^{m}\right\|_{Y_{S}(\Omega)} \leq \kappa_{0}^{-1}\left\|\omega_{0}\right\|_{L^{2}(\Omega)}\|\mathcal{I}\|_{\mathcal{L}\left(Y_{S}(\Omega), L^{2}(\Omega)\right)} .
$$

With this majoration, we deduce from (18) that

$$
\begin{aligned}
\left\|\boldsymbol{u}^{m}\right\|_{\boldsymbol{V}_{S}(\Omega)} \leq & \mu_{0}^{-1}\left\|\pi^{0}\right\|_{L^{2}(S)}\left\|\gamma_{S}\right\|_{\mathcal{L}\left(\boldsymbol{V}_{S}(\Omega), \mathbf{L}^{2}(S)\right)}+\mu_{0}^{-1}\left\|g_{0}\right\|_{L^{2}(\Omega)}\|\mathcal{I}\|_{\mathcal{L}\left(\boldsymbol{V}_{S}(\Omega), \mathbf{L}^{2}(\Omega)\right)} \\
& +M \mu_{0}^{-1} \kappa_{0}^{-2}\left\|\omega_{0}\right\|_{L^{2}(\Omega)}^{2}\|\mathcal{I}\|_{\mathcal{L}\left(\gamma_{S}(\Omega), L^{4}(\Omega)\right)}^{2}\|\mathcal{I}\|_{\mathcal{L}\left(\boldsymbol{V}_{S}(\Omega), \boldsymbol{L}^{2}(\Omega)\right)}\|\mathcal{I}\|_{\mathcal{L}\left(\gamma_{S}(\Omega), L^{2}(\Omega)\right)}^{2}
\end{aligned}
$$

Furthermore, since $\left\{\boldsymbol{v}^{j}\right\}_{j=1}^{\infty}$ is an orthonormal basis of the space $\boldsymbol{V}_{S}(\Omega)$ and $\left\{\eta^{j}\right\}_{j=1}^{\infty}$ is an orthonormal basis of the space $Y_{S}(\Omega)$, from (13) it follows that

$$
\left\|\boldsymbol{u}^{m}\right\|_{\boldsymbol{V}_{S}(\Omega)}^{2}=\sum_{j=1}^{m} a_{m j}^{2}, \quad\left\|\theta^{m}\right\|_{Y_{S}(\Omega)}^{2}=\sum_{j=1}^{m} b_{m j}^{2}
$$

Taking into account (20) and (21), we obtain

$$
\begin{aligned}
\sum_{j=1}^{m} a_{m j}^{2} \leq & \left\{\mu_{0}^{-1}\left\|\pi^{0}\right\|_{L^{2}(S)}\left\|\gamma_{S}\right\|_{\mathcal{L}\left(V_{S}(\Omega), L^{2}(S)\right)}+\mu_{0}^{-1}\left\|g_{0}\right\|_{L^{2}(\Omega)}\|\mathcal{I}\|_{\mathcal{L}\left(V_{S}(\Omega), L^{2}(\Omega)\right)}\right. \\
& \left.+M \mu_{0}^{-1} \mathcal{K}_{0}^{-2}\left\|\omega_{0}\right\|_{L^{2}(\Omega)}^{2}\|\mathcal{I}\|_{\mathcal{L}\left(Y_{S}(\Omega), L^{4}(\Omega)\right)}^{2}\|\mathcal{I}\|_{\mathcal{L}\left(V_{S}(\Omega), L^{2}(\Omega)\right)}\|\mathcal{I}\|_{\mathcal{L}\left(Y_{S}(\Omega), L^{2}(\Omega)\right)}^{2}\right\}^{2}, \\
\sum_{j=1}^{m} b_{m j}^{2} \leq & \kappa_{0}^{-2}\left\|\omega_{0}\right\|_{L^{2}(\Omega)}^{2}\|\mathcal{I}\|_{\mathcal{L}\left(Y_{S}(\Omega), L^{2}(\Omega)\right)}^{2} .
\end{aligned}
$$

Note that these estimates do not depend on $m \in \mathbb{N}$ as well as $\lambda \in[0,1]$. Therefore, we can apply Proposition 1 to justify the solvability of problem (11)-(13). Here, of course, we used Proposition 2 and conditions (C2)-(C5) to establish the continuity of the corresponding mappings.

Let $\left(\widetilde{a}_{m 1}, \ldots, \widetilde{a}_{m m}, \widetilde{b}_{m 1}, \ldots, \widetilde{b}_{m m}\right)$ be a solution to (11)-(13) with $\lambda=1$. Setting

$$
\widetilde{\boldsymbol{u}}^{m} \stackrel{\text { def }}{=} \sum_{j=1}^{m} \widetilde{a}_{m j} \boldsymbol{v}^{j}, \quad \widetilde{\theta}^{m} \stackrel{\text { def }}{=} \sum_{j=1}^{m} \widetilde{b}_{m j} \eta^{j},
$$

we have 


$$
\begin{array}{r}
\int_{\Omega} \mu\left(\widetilde{\theta}^{m}\right) \boldsymbol{D}\left(\widetilde{\boldsymbol{u}}^{m}\right): \boldsymbol{D}\left(\boldsymbol{v}^{j}\right) d x+\int_{S} \pi^{0}\left(\boldsymbol{v}^{j} \cdot \mathbf{n}\right) d \sigma=\int_{\Omega} f\left(\boldsymbol{x}, \widetilde{\theta}^{m}\right) \cdot \boldsymbol{v}^{j} \boldsymbol{d} \boldsymbol{x}, \quad j=1, \ldots, m, \\
\sum_{i=1}^{d} \int_{\Omega} \widetilde{u}_{i}^{m} \frac{\partial \widetilde{\theta}^{m}}{\partial x_{i}} \eta^{j} d x+\int_{\Omega} \kappa\left(\widetilde{\theta}^{m}\right) \nabla \widetilde{\theta}^{m} \cdot \nabla \eta^{j} d x+\alpha \int_{\partial \Omega \backslash S} \widetilde{\theta}^{m} \eta^{j} d \sigma=\int_{\Omega} \omega\left(\boldsymbol{x}, \widetilde{\theta}^{m}\right) \eta^{j} d x, \\
j=1, \ldots, m .
\end{array}
$$

Consider the sequence $\left\{\left(\widetilde{\boldsymbol{u}}^{m}, \widetilde{\theta}^{m}\right)\right\}_{m=1}^{\infty}$. It is clear that estimate (21) remains valid if we replace $\boldsymbol{u}^{m}$ with $\widetilde{\boldsymbol{u}}^{m}$. Consequently, the sequence $\left\{\widetilde{\boldsymbol{u}}^{m}\right\}_{m=1}^{\infty}$ is bounded in the space $\boldsymbol{V}_{S}(\Omega)$. Likewise, taking into account estimate (20), we see that the sequence $\left\{\widetilde{\theta}^{m}\right\}_{m=1}^{\infty}$ is bounded in the space $Y_{S}(\Omega)$. Therefore, there exists a pair $\left(\widetilde{\boldsymbol{u}}^{0}, \widetilde{\theta}^{0}\right) \in \boldsymbol{V}_{S}(\Omega) \times Y_{S}(\Omega)$ such that $\widetilde{\boldsymbol{u}}^{m_{k}}$ converges to $\widetilde{\boldsymbol{u}}^{0}$ weakly in $\boldsymbol{V}_{S}(\Omega)$ and $\widetilde{\theta}^{m_{k}}$ converges to $\widetilde{\theta}^{0}$ weakly in $Y_{S}(\Omega)$, for some subsequence $m_{k} \rightarrow \infty$ as $k \rightarrow \infty$. Without loss of generality, it can be assumed that

$$
\begin{gathered}
\widetilde{\boldsymbol{u}}^{m} \rightarrow \widetilde{\boldsymbol{u}}^{0} \text { weakly in } \boldsymbol{V}_{S}(\Omega) \text { as } m \rightarrow \infty, \\
\widetilde{\theta}^{m} \rightarrow \widetilde{\theta}^{0} \text { weakly in } Y_{S}(\Omega) \text { as } m \rightarrow \infty .
\end{gathered}
$$

Since the trace operator $\gamma_{\partial \Omega}: H^{1}(\Omega) \rightarrow L^{2}(\partial \Omega)$ is compact (see [15] Section 2.6.2, Theorem 6.2), we have

$$
\left.\left.\widetilde{\theta}^{m}\right|_{\partial \Omega \backslash S} \rightarrow \widetilde{\theta}^{0}\right|_{\partial \Omega \backslash S} \text { strongly in } L^{2}(\partial \Omega \backslash S) \text { as } m \rightarrow \infty .
$$

Moreover, from (24) and (25) and the compactness theorem for the identity mapping $\mathcal{I}: H^{1}(\Omega) \rightarrow L^{4}(\Omega)$ (see [15] Section 2.6.1, Theorem 6.1), it follows that

$$
\begin{aligned}
& \widetilde{\boldsymbol{u}}^{m} \rightarrow \widetilde{\boldsymbol{u}}^{0} \text { strongly in } L^{4}(\Omega) \text { as } m \rightarrow \infty, \\
& \widetilde{\theta}^{m} \rightarrow \widetilde{\theta}^{0} \text { strongly in } L^{4}(\Omega) \text { as } m \rightarrow \infty .
\end{aligned}
$$

Using (24)-(28) and Proposition 2, we can pass to the limit $m \rightarrow \infty$ in (22) and (23); this gives

$$
\begin{gathered}
\int_{\Omega} \mu\left(\widetilde{\theta}^{0}\right) \boldsymbol{D}\left(\widetilde{\boldsymbol{u}}^{0}\right): \boldsymbol{D}\left(\boldsymbol{v}^{j}\right) d \boldsymbol{x}+\int_{S} \pi^{0}\left(\boldsymbol{v}^{j} \cdot \mathbf{n}\right) d \sigma=\int_{\Omega} f\left(x, \widetilde{\theta}^{0}\right) \cdot \boldsymbol{v}^{j} d x, \\
\sum_{i=1}^{d} \int_{\Omega} \widetilde{u}_{i}^{0} \frac{\partial \widetilde{\theta}^{0}}{\partial x_{i}} \eta^{j} d x+\int_{\Omega} \kappa\left(\widetilde{\theta}^{0}\right) \nabla \widetilde{\theta}^{0} \cdot \nabla \eta^{j} d x+\alpha \int_{\partial \Omega \backslash S} \widetilde{\theta}^{0} \eta^{j} d \sigma=\int_{\Omega} \omega\left(\boldsymbol{x}, \widetilde{\theta}^{0}\right) \eta^{j} d x,
\end{gathered}
$$

for each $j \in \mathbb{N}$. Because $\left\{\boldsymbol{v}^{j}\right\}_{j=1}^{\infty}$ is a basis of $\boldsymbol{V}_{S}(\Omega)$ and $\left\{\eta^{j}\right\}_{j=1}^{\infty}$ is a basis of $Y_{S}(\Omega)$, equalities (29) and (30) remain valid if we replace $v^{j}$ and $\eta^{j}$ with arbitrary vector function $v \in V_{S}(\Omega)$ and function $\eta \in Y_{S}(\Omega)$, respectively:

$$
\begin{gathered}
\int_{\Omega} \mu\left(\widetilde{\theta}^{0}\right) \boldsymbol{D}\left(\widetilde{\boldsymbol{u}}^{0}\right): \boldsymbol{D}(\boldsymbol{v}) d \boldsymbol{x}+\int_{S} \pi^{0}(\boldsymbol{v} \cdot \mathbf{n}) d \sigma=\int_{\Omega} \boldsymbol{f}\left(\boldsymbol{x}, \widetilde{\theta}^{0}\right) \cdot \boldsymbol{v} \boldsymbol{d} \boldsymbol{x}, \\
\sum_{i=1}^{d} \int_{\Omega} \widetilde{u}_{i}^{0} \frac{\partial \widetilde{\theta}^{0}}{\partial x_{i}} \eta \boldsymbol{d x}+\int_{\Omega} \kappa\left(\widetilde{\theta}^{0}\right) \nabla \widetilde{\theta}^{m} \cdot \nabla \eta \boldsymbol{d} \boldsymbol{x}+\alpha \int_{\partial \Omega \backslash S} \widetilde{\theta}^{0} \eta d \sigma=\int_{\Omega} \omega\left(\boldsymbol{x}, \widetilde{\theta}^{0}\right) \eta \boldsymbol{d} x .
\end{gathered}
$$

Thus, we established that $\left(\widetilde{\boldsymbol{u}}^{0}, \widetilde{\theta}^{0}, \pi^{0}, 0\right)$ is an admissible quadruplet to problem (1) and hence $\mathfrak{M}\left(\mathbb{U}_{1}, \mathbb{U}_{2}\right) \neq \varnothing$.

Now, consider a sequence $\left\{\left(\widehat{\boldsymbol{u}}^{s}, \widehat{\theta}^{s}, \widehat{\pi}^{s}, \widehat{\zeta}^{s}\right)\right\}_{s=1}^{\infty} \subset \mathfrak{M}\left(\mathbb{U}_{1}, \mathbb{U}_{2}\right)$ such that

$$
\lim _{s \rightarrow \infty} J\left(\widehat{\boldsymbol{u}}^{s}, \widehat{\theta}^{s}, \widehat{\pi}^{s}, \widehat{\zeta}^{s}\right)=\inf _{(\boldsymbol{u}, \theta, \pi, \zeta) \in \mathfrak{M}\left(\mathbb{U}_{1}, \mathbb{U}_{2}\right)} J(\boldsymbol{u}, \theta, \pi, \zeta) .
$$


Owing to coercivity condition (C9), we deduce from (31) that the set $\left\{\left(\widehat{\boldsymbol{u}}^{s}, \widehat{\theta}^{s}, \widehat{\pi}^{s}, \widehat{\zeta}^{s}\right)\right\}_{s=1}^{\infty}$ is bounded in the space $V_{S}(\Omega) \times H^{1}(\Omega) \times L^{2}(S) \times L^{2}(S)$. Hence, there exists a subsequence $\left\{s_{k}\right\}_{k=1}^{\infty}$ such that

$$
\begin{gathered}
\widehat{\boldsymbol{u}}^{s_{k}} \rightarrow \boldsymbol{u}_{*} \text { weakly in } \boldsymbol{V}_{S}(\Omega) \text { as } k \rightarrow \infty, \\
\widehat{\theta}^{s_{k}} \rightarrow \theta_{*} \text { weakly in } \gamma_{S}(\Omega) \text { as } k \rightarrow \infty, \\
\widehat{\pi}^{s_{k}} \rightarrow \pi_{*} \text { weakly in } L^{2}(S) \text { as } k \rightarrow \infty, \\
\widehat{\zeta}^{s_{k}} \rightarrow \zeta_{*} \text { weakly in } L^{2}(S) \text { as } k \rightarrow \infty,
\end{gathered}
$$

for some quadruplet $\left(\boldsymbol{u}_{*}, \theta_{*}, \pi_{*}, \zeta_{*}\right) \in \boldsymbol{V}_{S}(\Omega) \times H^{1}(\Omega) \times L^{2}(S) \times L^{2}(S)$.

The inclusion $\left\{\left(\widehat{\boldsymbol{u}}^{s_{k}}, \widehat{\theta}^{s_{k}}, \widehat{\pi}^{s_{k}}, \widehat{\zeta}^{s_{k}}\right)\right\}_{k=1}^{\infty} \subset \mathfrak{M}\left(\mathbb{U}_{1}, \mathbb{U}_{2}\right)$ implies that

$$
\begin{gathered}
\int_{\Omega} \mu\left(\widehat{\theta}^{s_{k}}\right) \boldsymbol{D}\left(\widehat{\boldsymbol{u}}^{s_{k}}\right): \boldsymbol{D}(\boldsymbol{v}) d \boldsymbol{x}+\int_{S} \widehat{\pi}^{s_{k}}(\boldsymbol{v} \cdot \mathbf{n}) d \sigma=\int_{\Omega} \boldsymbol{f}\left(\boldsymbol{x}, \widehat{\theta}^{s_{k}}\right) \cdot \boldsymbol{v} \boldsymbol{d} \boldsymbol{x}, \quad \forall \boldsymbol{v} \in \boldsymbol{V}_{S}(\Omega), \\
\sum_{i=1}^{d} \int_{\Omega} \widehat{u}_{i}^{s_{k}} \frac{\partial \widehat{\theta}^{s_{k}}}{\partial x_{i}} \eta \boldsymbol{d} \boldsymbol{x}+\int_{\Omega} \kappa\left(\widehat{\theta}^{s_{k}}\right) \nabla \widehat{\theta}^{s_{k}} \cdot \nabla \eta \boldsymbol{d} \boldsymbol{x}+\alpha \int_{\partial \Omega \backslash S} \widehat{\theta}^{s_{k}} \eta d \sigma=\int_{\Omega} \omega\left(\boldsymbol{x}, \widehat{\theta}^{s_{k}}\right) \eta \boldsymbol{d} \boldsymbol{x}, \quad \forall \eta \in Y_{S}(\Omega),
\end{gathered}
$$

from which, by (32)-(35), one can derive the following equalities:

$$
\begin{gathered}
\int_{\Omega} \mu\left(\theta_{*}\right) \boldsymbol{D}\left(\boldsymbol{u}_{*}\right): \boldsymbol{D}(\boldsymbol{v}) d x+\int_{S} \pi_{*}(\boldsymbol{v} \cdot \mathbf{n}) d \sigma=\int_{\Omega} f\left(x, \theta_{*}\right) \cdot \boldsymbol{v} d x, \quad \forall \boldsymbol{v} \in \boldsymbol{V}_{S}(\Omega), \\
\sum_{i=1}^{d} \int_{\Omega} u_{* i} \frac{\partial \theta_{*}}{\partial x_{i}} \eta \boldsymbol{d} \boldsymbol{x}+\int_{\Omega} \kappa\left(\theta_{*}\right) \nabla \theta_{*} \cdot \nabla \eta \boldsymbol{d} \boldsymbol{x}+\alpha \int_{\partial \Omega \backslash S} \theta_{*} \eta d \sigma=\int_{\Omega} \omega\left(\boldsymbol{x}, \theta_{*}\right) \eta \boldsymbol{d} \boldsymbol{x}, \quad \forall \eta \in Y_{S}(\Omega) .
\end{gathered}
$$

Note that

$$
\widehat{\zeta}^{s_{k}}=\left.\left.\widehat{\theta}^{s_{k}}\right|_{S} \rightarrow \theta_{*}\right|_{S} \text { strongly in } L^{2}(S) \text { as } k \rightarrow \infty .
$$

Comparing this convergence with (35), we conclude that $\left.\theta_{*}\right|_{S}=\zeta_{*}$. Moreover, since $\left\{\widehat{\zeta}^{s_{k}}\right\}_{k=1}^{\infty} \subset \mathbb{U}_{2}$ and the set $\mathbb{U}_{2}$ is closed in the space $L^{2}(S)$, we see that the function $\zeta_{*}$ belongs to $\mathbb{U}_{2}$. Next, taking into account condition (C6), the inclusion $\left\{\hat{\pi}^{s_{k}}\right\}_{k=1}^{\infty} \subset \mathbb{U}_{1}$, and convergence (34), we get $\pi_{*} \in \mathbb{U}_{1}$. Thus, $\left(\boldsymbol{u}_{*}, \theta_{*}, \pi_{*}, \zeta_{*}\right) \in \mathfrak{M}\left(\mathbb{U}_{1}, \mathbb{U}_{2}\right)$.

In addition, by condition (C8) and (31)-(35), we can obtain

$$
\begin{aligned}
J\left(\boldsymbol{u}_{*}, \theta_{*}, \pi_{*}, \zeta_{*}\right) & \leq \liminf _{k \rightarrow \infty} J\left(\widehat{\boldsymbol{u}}^{s_{k}}, \widehat{\theta}^{s_{k}}, \widehat{\pi}^{s_{k}}, \widehat{\zeta}^{s_{k}}\right) \\
& =\inf _{(\boldsymbol{u}, \theta, \pi, \zeta) \in \mathfrak{M}\left(\mathbb{U}_{1}, \mathbb{U}_{2}\right)} J(\boldsymbol{u}, \theta, \pi, \zeta) .
\end{aligned}
$$

This means that the quadruplet $\left(\boldsymbol{u}_{*}, \theta_{*}, \pi_{*}, \zeta_{*}\right)$ belongs to the set $\mathfrak{M}_{\text {opt }}\left(\mathbb{U}_{1}, \mathbb{U}_{2}\right)$, and hence $\mathfrak{M}_{\text {opt }}\left(\mathbb{U}_{1}, \mathbb{U}_{2}\right) \neq \varnothing$. Therefore, we have shown that optimal control problem (1) is solvable in the sense of Definition 2.

We now turn to proving assertion (b). Assume the converse. Then, there exists a subsequence $\left\{n_{k}\right\}_{k=1}^{\infty}$ such that

$$
\lim _{k \rightarrow \infty} \Phi\left(\mathbb{U}_{1}^{n_{k}}, \mathbb{U}_{2}^{n_{k}}\right)<\Phi\left(\mathbb{U}_{1}, \mathbb{U}_{2}\right)
$$

Consider a sequence $\left\{\left(\boldsymbol{u}_{*}^{n_{k}}, \theta_{*}^{n_{k}}, \pi_{*}^{n_{k}}, \zeta_{*}^{n_{k}}\right)\right\}_{k=1}^{\infty}$ such that

$$
\left(\boldsymbol{u}_{*}^{n_{k}}, \theta_{*}^{n_{k}}, \pi_{*}^{n_{k}}, \zeta_{*}^{n_{k}}\right) \in \mathfrak{M}_{\mathrm{opt}}\left(\mathbb{U}_{1}^{n_{k}}, \mathbb{U}_{2}^{n_{k}}\right), \quad \forall k \in \mathbb{N} .
$$

By Definition 1, we get 


$$
\begin{array}{r}
\int_{\Omega} \mu\left(\theta_{*}^{n_{k}}\right) \boldsymbol{D}\left(\boldsymbol{u}_{*}^{n_{k}}\right): \boldsymbol{D}(\boldsymbol{v}) d x+\int_{S} \pi_{*}^{n_{k}}(\boldsymbol{v} \cdot \mathbf{n}) d \sigma=\int_{\Omega} f\left(x, \theta_{*}^{n_{k}}\right) \cdot \boldsymbol{v} d \boldsymbol{x}, \quad \forall \boldsymbol{v} \in \boldsymbol{V}_{S}(\Omega), \\
\sum_{i=1}^{d} \int_{\Omega} u_{* i}^{n_{k}} \frac{\partial \theta_{*}^{n_{k}}}{\partial x_{i}} \eta \boldsymbol{d} x+\int_{\Omega} \kappa\left(\theta_{*}^{n_{k}}\right) \nabla \theta_{*}^{n_{k}} \cdot \nabla \eta \boldsymbol{d} x+\alpha \int_{\partial \Omega \backslash S} \theta_{*}^{n_{k}} \eta d \sigma=\int_{\Omega} \omega\left(\boldsymbol{x}, \theta_{*}^{n_{k}}\right) \eta \boldsymbol{d} x, \\
\forall \eta \in Y_{S}(\Omega) .
\end{array}
$$

Moreover, by Definition 3, we have

$$
\Phi\left(\mathbb{U}_{1}^{n_{k}}, \mathbb{U}_{2}^{n_{k}}\right)=J\left(\boldsymbol{u}_{*}^{n_{k}}, \theta_{*}^{n_{k}}, \pi_{*}^{n_{k}}, \zeta_{*}^{n_{k}}\right), \quad \forall k \in \mathbb{N} .
$$

It follows from (36) and (39) that there exists a number $k_{0}$ such that

$$
J\left(\boldsymbol{u}_{*}^{n_{k}}, \theta_{*}^{n_{k}}, \pi_{*}^{n_{k}}, \zeta_{*}^{n_{k}}\right)<\Phi\left(\mathbb{U}_{1}, \mathbb{U}_{2}\right)
$$

for each $k \geq k_{0}$. Hence,

$$
\left\{\left(\boldsymbol{u}_{*}^{n_{k}}, \theta_{*}^{n_{k}}, \pi_{*}^{n_{k}}, \zeta_{*}^{n_{k}}\right)\right\}_{k=k_{0}}^{\infty} \subset \mathcal{G}_{\left|\Phi\left(\mathbb{U}_{1}, \mathbb{U}_{2}\right)\right|} \cdot
$$

In view of condition (C9), the set $\mathcal{G}_{\left|\Phi\left(\mathbb{U}_{1}, \mathbb{U}_{2}\right)\right|}$ is bounded in $\boldsymbol{V}_{S}(\Omega) \times H^{1}(\Omega) \times L^{2}(S) \times L^{2}(S)$. Therefore, without loss of generality, it can be assumed that

$$
\begin{gathered}
\boldsymbol{u}_{*}^{n_{k}} \rightarrow \boldsymbol{u}_{*}^{0} \text { weakly in } \boldsymbol{V}_{S}(\Omega) \text { as } k \rightarrow \infty, \\
\theta_{*}^{n_{k}} \rightarrow \theta_{*}^{0} \text { weakly in } Y_{S}(\Omega) \text { as } k \rightarrow \infty, \\
\pi_{*}^{n_{k}} \rightarrow \pi_{*}^{0} \text { weakly in } L^{2}(S) \text { as } k \rightarrow \infty, \\
\zeta_{*}^{n_{k}} \rightarrow \zeta_{*}^{0} \text { weakly in } L^{2}(S) \text { as } k \rightarrow \infty,
\end{gathered}
$$

for some quadruplet $\left(\boldsymbol{u}_{*}^{0}, \theta_{*}^{0}, \pi_{*}^{0}, \zeta_{*}^{0}\right) \in \boldsymbol{V}_{S}(\Omega) \times H^{1}(\Omega) \times L^{2}(S) \times L^{2}(S)$.

Besides, since both the identity mapping $\mathcal{I}: H^{1}(\Omega) \rightarrow L^{4}(\Omega)$ and the trace operator $\gamma_{\partial \Omega}: H^{1}(\Omega) \rightarrow L^{2}(\partial \Omega)$ are compact, the following convergences hold:

$$
\begin{gathered}
\boldsymbol{u}_{*}^{n_{k}} \rightarrow \boldsymbol{u}_{*}^{0} \text { strongly in } L^{4}(\Omega) \text { as } k \rightarrow \infty, \\
\theta_{*}^{n_{k}} \rightarrow \theta_{*}^{0} \text { strongly in } L^{4}(\Omega) \text { as } k \rightarrow \infty, \\
\zeta_{*}^{n_{k}}=\left.\left.\theta_{*}^{n_{k}}\right|_{S} \rightarrow \theta_{*}^{0}\right|_{S} \text { strongly in } L^{2}(S) \text { as } k \rightarrow \infty .
\end{gathered}
$$

Comparing (43) with (46), one can infer that $\left.\theta_{*}^{0}\right|_{S}=\zeta_{*}^{0}$ and

$$
\zeta_{*}^{n_{k}} \rightarrow \zeta_{*}^{0} \text { strongly in } L^{2}(S) \text { as } k \rightarrow \infty .
$$

In view of (10), there exist sequences $\left\{\bar{\pi}^{n_{k}}\right\}_{k=1}^{\infty} \subset \mathbb{U}_{1}$ and $\left\{\bar{\zeta}^{n_{k}}\right\}_{k=1}^{\infty} \subset \mathbb{U}_{2}$ such that

$$
\lim _{k \rightarrow \infty}\left\|\pi_{*}^{n_{k}}-\bar{\pi}^{n_{k}}\right\|_{L^{2}(S)}=0, \quad \lim _{k \rightarrow \infty}\left\|\zeta_{*}^{n_{k}}-\bar{\zeta}^{n_{k}}\right\|_{L^{2}(S)}=0 .
$$

From (42), (47), and (48), it follows that

$$
\begin{aligned}
& \bar{\pi}^{n_{k}} \rightarrow \pi_{*}^{0} \text { weakly in } L^{2}(S) \text { as } k \rightarrow \infty, \\
& \bar{\zeta}^{n_{k}} \rightarrow \zeta_{*}^{0} \text { strongly in } L^{2}(S) \text { as } k \rightarrow \infty,
\end{aligned}
$$

and, by conditions (C6) and (C7), we get $\left(\pi_{*}^{0}, \zeta_{*}^{0}\right) \in \mathbb{U}_{1} \times \mathbb{U}_{2}$. 
Next, taking into account the convergence results (40)-(42), (44), and (45), we pass to the limit $k \rightarrow \infty$ in equalities (37) and (38) and obtain

$$
\begin{gathered}
\int_{\Omega} \mu\left(\theta_{*}^{0}\right) \boldsymbol{D}\left(\boldsymbol{u}_{*}^{0}\right): \boldsymbol{D}(\boldsymbol{v}) d \boldsymbol{x}+\int_{S} \pi_{*}^{0}(\boldsymbol{v} \cdot \mathbf{n}) d \sigma=\int_{\Omega} \boldsymbol{f}\left(\boldsymbol{x}, \theta_{*}^{0}\right) \cdot \boldsymbol{v} \boldsymbol{d} \boldsymbol{x}, \quad \forall \boldsymbol{v} \in \boldsymbol{V}_{S}(\Omega), \\
\sum_{i=1}^{d} \int_{\Omega} u_{* i}^{0} \frac{\partial \theta_{*}^{0}}{\partial x_{i}} \eta \boldsymbol{d} \boldsymbol{x}+\int_{\Omega} \kappa\left(\theta_{*}^{0}\right) \nabla \theta_{*}^{0} \cdot \nabla \eta \boldsymbol{d} \boldsymbol{x}+\alpha \int_{\partial \Omega \backslash S} \theta_{*}^{0} \eta d \sigma=\int_{\Omega} \omega\left(\boldsymbol{x}, \theta_{*}^{0}\right) \eta \boldsymbol{d} \boldsymbol{x}, \quad \forall \eta \in Y_{S}(\Omega) .
\end{gathered}
$$

Therefore, we have established that the quadruplet $\left(\boldsymbol{u}_{*}^{0}, \theta_{*}^{0}, \pi_{*}^{0}, \zeta_{*}^{0}\right)$ belongs to the set $\mathfrak{M}\left(\mathbb{U}_{1}, \mathbb{U}_{2}\right)$. This is a key point of the proof of assertion (b). Indeed, using condition (C8) and (39)-(43), we can deduce the following relations:

$$
\begin{aligned}
\Phi\left(\mathbb{U}_{1}, \mathbb{U}_{2}\right) & =\inf _{(\boldsymbol{u}, \theta, \pi, \zeta) \in \mathfrak{M}\left(\mathbb{U}_{1}, \mathbb{U}_{2}\right)} J(\boldsymbol{u}, \theta, \pi, \zeta) \\
& \leq J\left(\boldsymbol{u}_{*}^{0}, \theta_{*}^{0}, \pi_{*}^{0}, \zeta_{*}^{0}\right) \\
& \leq \liminf _{k \rightarrow \infty} J\left(\boldsymbol{u}_{*}^{n_{k}}, \theta_{*}^{n_{k}}, \pi_{*}^{n_{k}}, \zeta_{*}^{n_{k}}\right) \\
& =\lim _{k \rightarrow \infty} \Phi\left(\mathbb{U}_{1}^{n_{k}}, \mathbb{U}_{2}^{n_{k}}\right),
\end{aligned}
$$

thus contradicting inequality (36). This contradiction concludes the proof.

\section{Concluding Remarks}

In this study, we considered an optimal control problem for the system of nonlinear equations describing steady non-isothermal creeping flows of an incompressible fluid through a locally Lipschitz bounded domain. Using the discussion presented above, we established the existence of weak solutions that minimize a given cost functional. For the considered control system, we also introduced the concept of the marginal function and proved that this function is lower semi-continuous. This means that it is impossible to achieve a significant improvement in the optimal value of the cost functional by small changes in the set of admissible controls.

Author Contributions: Conceptualization, E.S.B.; methodology, E.S.B.; writing—original draft, E.S.B. and A.A.D.; writing-review and editing, M.A.A.

Funding: This research received no external funding.

Conflicts of Interest: The authors declare no conflict of interest.

\section{References}

1. Lee, H.C.; Imanuvilov, O.Y. Analysis of optimal control problems for the 2-D stationary Boussinesq equations. J. Math. Anal. Appl. 2000, 242, 191-211. [CrossRef]

2. Lee, H.-C.; Shin, B.C. Piecewise optimal distributed controls for 2D Boussinesq equations. Math. Meth. Appl. Sci. 2000, 23, 227-254. [CrossRef]

3. Li, S.; Wang, G. The time optimal control of the Boussinesq equations. Numer. Funct. Anal. Optim. 2003, 24, 163-180. [CrossRef]

4. Trenchea, C. Periodic optimal control of the Boussinesq equation. Nonlinear Anal. 2003, 53, 81-96. [CrossRef]

5. Lee, H.-C.; Kim, S. Finite element approximation and computations of optimal Dirichlet boundary control problems for the Boussinesq equations. J. Korean Math. Soc. 2004, 41, 681-715. [CrossRef]

6. Guerrero, S. Local exact controllability to the trajectories of the Boussinesq system. Ann. Inst. Henri Poincaré Anal. Non Linéaire 2006, 23, 29-61. [CrossRef]

7. Hinze, M.; Matthes, U. Optimal and model predictive control of the Boussinesq approximation. In Control of Coupled Partial Differential Equations; Kunisch, K., Leugering, G., Sprekels, J., Tröltzsch, F., Eds.; International Series of Numerical Mathematics; Birkhäuser Verlag AG: Basel, Switzerland, 2007; Volume 155, pp. 149-174, ISBN 978-3-7643-7720-5. 
8. Korotkii, A.I.; Kovtunov, D.A. Optimal boundary control of a system describing thermal convection. Proc. Steklov Inst. Math. 2011, 272 (Suppl. 1), S74-S100. [CrossRef]

9. Alekseev, G.V. Solvability of stationary boundary control problems for heat convection equations. Sib. Math. J. 1998, 39, 844-858. [CrossRef]

10. Fursikov, A.V.; Imanuvilov, O.Y. Exact controllability of the Navier-Stokes and Boussinesq equations. Russ. Math. Surv. 1999, 54, 565-618. [CrossRef]

11. Alekseev, G.V. Solvability of inverse extremal problems for stationary heat and mass transfer equations. Sib. Math. J. 2001, 42, 811-827. [CrossRef]

12. Alekseev, G.V.; Tereshko, D.A. Boundary control problems for stationary equations of heat convection. In New Directions in Mathematical Fluid Mechanics; Fursikov, A.V., Galdi, G., Pukhnachev, V.V., Eds.; The Alexander V. Kazhikhov Memorial Volume; Birkhäuser Verlag: Basel, Switzerland, 2010; pp. 1-21, ISBN 978-3-0346-0151-1.

13. Alekseev, G.V.; Tereshko, D.A. Stability of optimal controls for the stationary Boussinesq equations. Int. J. Differ. Equ. 2011, 2011. [CrossRef]

14. Conca, C.; Murat, F.; Pironneau, O. The Stokes and Navier-Stokes equations with boundary conditions involving the pressure. Jpn. J. Math. 1994, 20, 279-318. [CrossRef]

15. Nečas, J. Direct Methods in the Theory of Elliptic Equations; Springer: Heidelberg, Germany, 2012.

16. Litvinov, V.G. Motion of a Nonlinear-Viscous Fluid; Nauka: Moscow, Russia, 1982.

17. Fursikov, A.V. Optimal Control of Distributed Systems: Theory and Applications; AMS: Providence, RI, USA, 2000.

18. O'Regan, D.; Cho, Y.J.; Chen, Y.Q. Topological Degree Theory and Applications; Taylor \& Francis Group: Boca Raton, FL, USA, 2006.

19. Krasnoselskii, M.A. Topological Methods in the Theory of Nonlinear Integral Equations; Pergamon Press: New York, NY, USA, 1964.

(C) 2019 by the authors. Licensee MDPI, Basel, Switzerland. This article is an open access article distributed under the terms and conditions of the Creative Commons Attribution (CC BY) license (http://creativecommons.org/licenses/by/4.0/). 